\title{
Use of n-Butyl 2 Cyanoacrylate without Lipiodol, Using a Modified Protocol in Gastric Variceal Bleed Management: Retrospective Analysis of 2299 Patients
}

\author{
Pankaj N. Desai ${ }^{1}$ Chintan N. Patel ${ }^{1}$ Mayank V. Kabrawala ${ }^{1} \quad$ Rajiv M. Mehta $^{2}$ \\ Subhash K. Nandwani ${ }^{2}$ Ritesh Prajapati ${ }^{2}$ Nisharg Patel ${ }^{2} \quad$ Krishna K. Parekh ${ }^{3}$
}

\footnotetext{
${ }^{1}$ Department of Endoscopy \& Endosonography, Surat Institute of Digestive Sciences (SIDS), Surat, India

${ }^{2}$ Department of Gastroenterology, Surat Institute of Digestive Sciences (SIDS), Surat, India

${ }^{3}$ Department of Clinical Research, Surat Institute of Digestive

Sciences (SIDS), Surat, India
}

J Digest Endosc:2020;11:187-192

\begin{abstract}
Address for correspondence Pankaj Desai, MS, FASGE, FAIGE, Department of Endoscopy \& Endosonography, Surat Institute of Digestive Sciences (SIDS), Majura Gate, Ring Road, Surat 395002, India (e-mail: drp_desai@hotmail.com).
\end{abstract}

\begin{abstract}
Keywords

- cyanoacrylate glue

- gastric varices

- variceal bleed

Aim To assess the feasibility and amount of cyanoacrylate glue that can be injected safely per session, complications, and long-term results in GOV1, GOV2 and IGV1 varices, using a modified method.

Method All patients from October 2008 to December 2019 presenting to us with variceal bleeding were included. We injected $0.5 \mathrm{~mL}$ of cyanoacrylate glue followed by $1.5 \mathrm{~mL}$ to $3 \mathrm{~mL}$ distilled water in all GOV 1, GOV 2 and IGV varices. Number of glues used were not restricted. Esophageal variceal ligation (EVL) was done for esophageal varices. Follow-up was done at 4 weeks and 3 to 6 months and analyzed.

Results 2299 patients received therapy. Male:female, $69 \%: 31 \%$. Causes of varices-cirrhosis (84.9\%) and extrahepatic portal vein obstruction (EHPVO) (15.1\%). Causes of cirrhosis-alcohol (38.4\%), nonalcoholic fatty liver disease (NAFLD) (29\%), hepatitis B (19.9\%), hepatitis C (10.1\%), Wilson's disease (1.8\%), and autoimmune diseases (0.9\%). As much as $41.1 \%$ had blood in stomach and $10.2 \%$ had active spurt on index endoscopy. As much as $14.5 \%$ required endotracheal intubation (active bleed [53.1\%], encephalopathy [40.5\%], respiratory distress [6.6\%]). As much as 30.9\% had GOV1 + GOV2, 1.5\% had GOV2 + IGV1, 21.3\% had GOV1 + IGV1 and $16.4 \%$ had GOV1 + GOV2 + IGV1. Hemostasis could be achieved in $99.2 \%$ on index endoscopy. A total of $18(0.8 \%)$ patients had uncontrolled bleed of whom $10(55.6 \%)$ had hepatocellular carcinoma. Ongoing bleed was treated with transjugular intrahepatic portosystemic shunt (TIPSS) (61.1\%) and endoscopic ultrasound (EUS)-guided coiling (38.8\%). Amount of glue required for gastric variceal obturation was 3.6 on index endoscopy, 1.6 on follow-up at 4 to 6 weeks, and 1.2 at 3 months follow up. Glue extrusion with ulcers were seen in $6.4 \%$ at 4 to 6 weeks and $4.3 \%$ at 3 months. Rebleed occurred in $2.1 \%$ and $0.7 \%$ patients before 4 weeks and 3 months follow-up, respectively. As much as $1.3 \%$ and $1.2 \%$ patients were lost to follow-up before 4 weeks and 3 months follow-up sessions, respectively. Complications include pulmonary aspiration in $0.9 \%$ and bleeding from ulcers $2.8 \%$. No evidence of pulmonary embolism was seen. In two endoscopic procedures, glue splashing into operator's eyes occurred. Incidents of needle getting stuck in the varix occurred in three patients.

Conclusions Our modified protocol for glue in gastric varices is safe and feasible, as it has less rebleed, no pulmonary embolism and less ulcers with glue extrusion as compared with available literature.
\end{abstract}

DOI https://doi.org/ 10.1055/s-0040-1716447 ISSN 0976-5042.
(C2020 Society of Gastrointestinal

Endoscopy of India
License terms

() (1) $\ominus \circledast$ 


\section{Introduction}

Varices are most superficial at the gastroesophageal (GE) junction and have the thinnest wall in that region; thus, variceal hemorrhage invariably occurs in that area. When the portal pressure increases above $12 \mathrm{~mm} \mathrm{Hg}$, the varix increases in size, with resultant decrease in wall thickness and increase in tension on the wall, which causes the varix to rupture. The direction of flow of blood in cases with portal hypertension is from below upward, that is, from the GE junction toward the esophagus. We hypothesized that obliterating the distal most portion of variceal flow will lead to less chances of incomplete variceal obliteration and rebleed. We followed Sarin's classification of gastric varices for management and analysis of our data ( - Fig. 1). All visible gastric varices in a patient with gastroesophageal variceal bleed were obliterated using cyanoacrylate glue on index and follow-up endoscopy.

Complications of glue include pulmonary embolism, stroke, multiorgan infarction via patent foramen ovale or arteriovenous pulmonary shunts, splenic infarction, splenic vein and portal vein thrombosis, and recurrent sepsis caused by embolized glue acting as a septic focus. As much as $4.3 \%$ rate of pulmonary embolism is quoted after glue injection. However, use of undiluted glue resulted in no cases of embolization in 170 patients. ${ }^{1}$ Transient pain and fever can occur in $90 \%$ of patients, A recent large retrospective study of 751 patients showed adverse events in 51 (6.8\%), 3.3\% with rebleeding caused by early extrusion of the glue cast, $1.3 \%$ with sepsis, $0.7 \%$ with embolism, and death in $4(0.53 \%)$. Lesser known complications are instrument damage and needle impaction in varix. ${ }^{2}$

Once injected in the varix, there is immediate solidification of the glue in 5 to 10 seconds. The mucosa overlying the varix
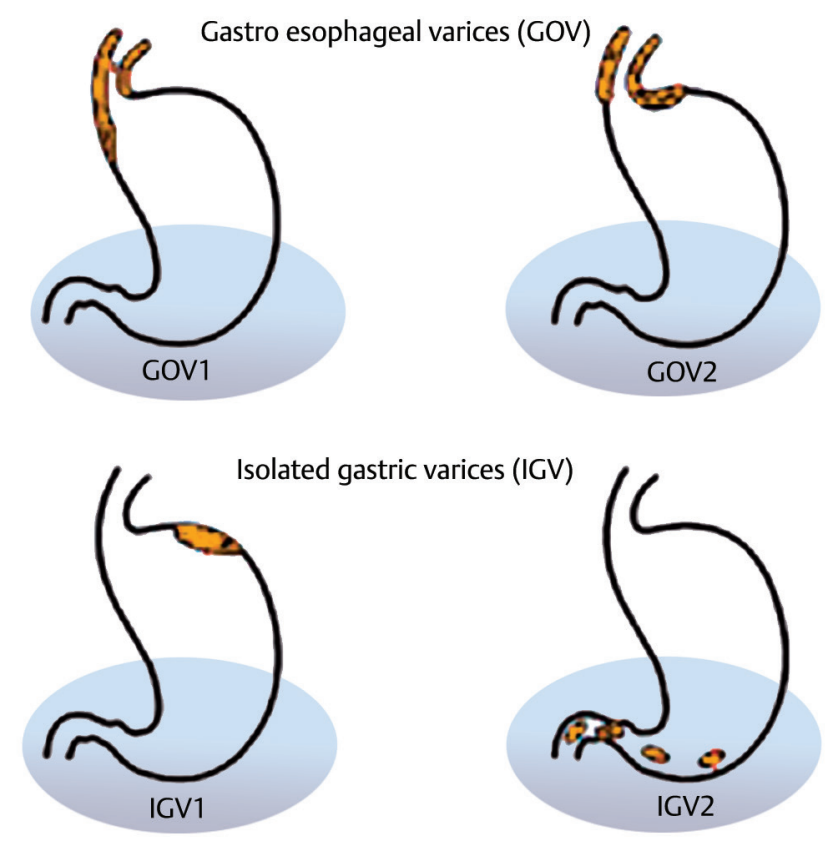

Fig. 1 Sarin's classification of gastric varices. then sloughs out, glue extrusion starts in about a months' time, and the glue is completely shed off in 3 months' time. Majority of the time there is evident fibrosis once the glue cast has been shed off, but occasionally there may be an ulcer at the site, and there is a likelihood that this ulcer can bleed. In conventional literature, glue has been used with lipiodol always in ratio of $1: 1$ or 1:1.6. In a few series where no lipiodol was used, the glue was used as $1 \mathrm{~mL}$ aliquots with $1 \mathrm{~mL}$ saline flush of the catheter. There is no hard data as to how much glue can be safely used in a single session of bleed. There is also sparse data of long-term efficacy and complications. Also, no standard guidelines are there for use of cyanoacrylate glue.

\section{Aim}

To assess the feasibility and amount of $n$-butyl 2 cyanoacrylate glue that can be injected safely per session, complications, and long-term results in GOV1, GOV2 and IGV1 varices, using a modified method.

\section{Method}

Retrospective analysis of prospectively collected data of all patients presenting to our hospital with variceal bleed from October 2008 to December 2019 were studied. The patients had variceal bleed secondary to portal hypertension, and etiologies were cirrhosis and extrahepatic portal vein obstruction (EHPVO).

Protocol followed for variceal bleed at our institution are as follows.

In a patient presenting with variceal bleed (ectopic varices were excluded), initial medical management was done as per existing guidelines. ${ }^{3}$ Single channel gastroscope was used in all cases. Index endoscopy (initial bleeding episode) was done within 12 hours of onset of bleed. All GOV 1 varices which extended more than $2 \mathrm{~cm}$ beyond the $\mathrm{Z}$ line into the gastric part, or in a hiatus hernia and were seen extending into the gastric cardia on retroflexion of the scope, GOV 2 varices and IGV1 varices were injected with glue. Bleeding source was either gastric, esophageal or unconfirmed. In our protocol, if the patient had both esophageal and gastric varices, then regardless of the source, whether the bleed is from esophageal or gastric varices, as a protocol, we injected the gastric varices first and then did esophageal variceal ligation (EVL) for the esophageal varices. Follow-up endoscopies were done at 4 to 6 weeks and 3-month intervals, and patients presenting with bleed till all esophageal and gastric varices were obliterated. Glue was injected on follow-up endoscopy in all patent gastric varices. EVL was done for esophageal varices as per established guidelines. We preferred a 23G needle (170 cm long) for all cases, as it was found to come out easily during retroflexion as compared with 21G. In addition, we did not find any difficulty in injecting glue from the 23G needle and found back bleeding from the glue injection site also less compared with $21 \mathrm{G}$, which we used very early in a few cases and which have not been included in this series. We used $0.5 \mathrm{~mL}$ aliquots for each 
varix regardless of the size of the varix, and this injection was followed by a rapid injection of $1.5 \mathrm{~mL}$ to $3 \mathrm{~mL}$ of distilled water. The amount of distilled water was calculated, depending on the size of the varix; smaller the varix, lesser was the amount. The end point of a single injection was till the varix bulged out and became engorged and solid. Maximum amount of distilled water in larger varices was $3 \mathrm{~mL}$. Further injection of distilled water was avoided to prevent glue embolization. For very large IGV varices and GOV 1 and 2 varices, we start injecting from the area of the varix arising from the gastric wall (at the far end from GE junction) and then coming to the area with maximum bulge at the apex which has the thinnest wall in the varix. The number of injections for each varix was not fixed. We injected as many glues as needed to completely solidify the varix. Any active spurt from the injection site was controlled with further glue injections. Hemostasis was defined as stopping of bleeding during endoscopy, with the varix becoming solid on palpation. Number of glue injections used per session, ulcerations at the glue site during subsequent sessions, and rebleed from that area were assessed. All patients received nonselective beta blocker (NSBB) if not contraindicated. Hence, the protocol included

1. Undiluted glue

2. Using $0.5 \mathrm{~mL}$ aliquots

3. Distilled water injected after glue injection, with endpoint being engorgement of varix or a total of $3 \mathrm{ml}$ injected during single puncture.

4. Number of injections for each varix was not restricted with the aim to completely solidify the varix.

The demographic data of all patients was noted. Causes of portal hypertension, causes of cirrhosis, active spurting from varices, risk of aspiration of gastric contents in emergency, need for endotracheal intubation in emergency, inability to cause hemostasis, complications, pulmonary embolism, and ischemia due to glue thrombus at any site and death were noted ( -Fig. 2).

\section{Results}

We included data of 2299 cases who came to us with variceal bleed from 2008 to 2019. Male (1593):female (706) ratio was $69 \%: 31 \%$. The major cause of variceal bleed was cirrhosis-1954 (84.9\%) followed by EHPVO 345 (15.1\%). Major cause of cirrhosis was alcohol-883 (38.4\%), nonalcoholic fatty liver disease (NAFLD) 666 (29\%), hepatitis B 457 (19.9\%), hepatitis C 232 (10.1\%) followed by Wilson's disease 41 (1.8\%), and autoimmune diseases $20(0.9 \%)$ (-Table 1).

As much as $63.1 \%$ had GOV1, 22.1\% had GOV2, $14.8 \%$ had IGV1 during index endoscopy. As much as $30.9 \%$ had GOV1 + GOV2, 1.5\% had GOV2 + IGV1, 21.3\% had GOV1 + IGV1 and 16.4\% had GOV1 + GOV2 + IGV1 (- Table 2). Of the IGV1, 8.2\% were $\mathrm{F} 1,43.8 \%$ were $\mathrm{F} 2$, and $47.9 \%$ were $\mathrm{F} 3$ ( - Table 3 ).

As much as $4 \%$ of patients did not receive NSBB due to contraindication or intolerance to it.

A mean number of $3.6(\times 0.5 \mathrm{~mL})$ glue was used (range 1 to 16) during index endoscopy. Hemostasis was achieved in 2281 (99.2\%) cases. Endotracheal intubation was needed in $333(14.5 \%)$ cases. The major reasons why intubation was done were massive active bleed 177 (53.1\%) with increased risk of aspiration, encephalopathy with bleed 135 (40.5\%), and respiratory distress 22 (6.6\%). We found blood in the stomach in 945 (41.1\%) cases. Actively bleeding varices were noted in $234(10.2 \%)$ cases ( - Table 4$)$.

Bleeding could not be controlled in $18(0.8 \%)$ cases. These patients with ongoing bleed were managed by transjugular intrahepatic portosystemic shunt (TIPSS) 11/18 (61.1\%)
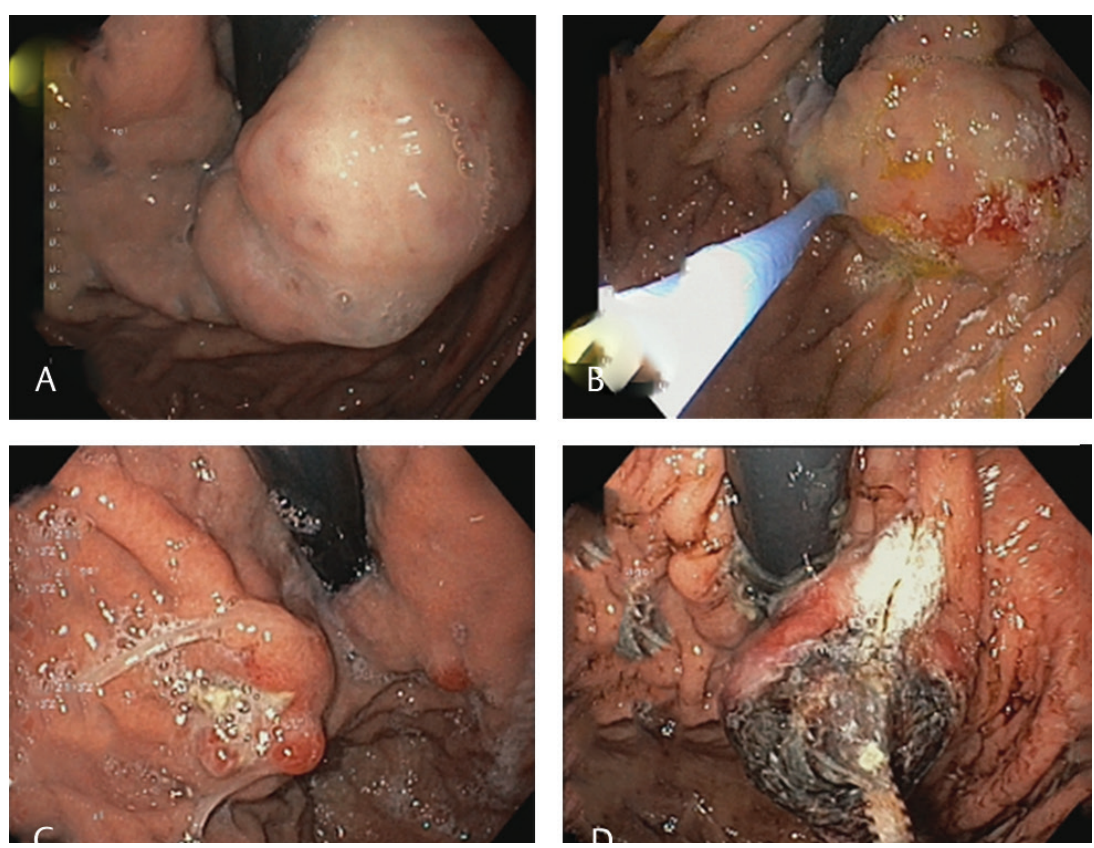

Fig. 2 Stages of glue injection: A-glue injection, B-solidified varix, C-glue with fibrosis, D-glue extrusion. 
Table 1 Demographic data and causes of varices and cirrhosis

\begin{tabular}{|l|l|}
\hline Clinical features & N (\%) \\
\hline Gender: Male & $1,593(69.3)$ \\
\hline Female & $706(30.7)$ \\
\hline Cause of Portal Hypertension & \\
\hline Cirrhosis & $1954(85)$ \\
\hline EHPVO & $345(15)$ \\
\hline Etiology of cirrhosis & \\
\hline Alcohol & $883(38.4)$ \\
\hline NAFLD & $666(29)$ \\
\hline Hepatitis B & $457(19.9)$ \\
\hline Hepatitis C & $232(10.1)$ \\
\hline Wilson's disease & $41(1.8)$ \\
\hline Autoimmune & $20(0.4)$ \\
\hline
\end{tabular}

Abbreviations: EHPVO, extrahepatic portal vein obstruction; NAFLD, nonalcoholic fatty liver disease.

Table 2 Classification of gastric varices

\begin{tabular}{|l|l|l|l|}
\hline & $\begin{array}{l}\text { Session 1 } \\
N(\%)\end{array}$ & $\begin{array}{l}\text { Session 2 } \\
N(\%)\end{array}$ & $\begin{array}{l}\text { Session 3 } \\
N(\%)\end{array}$ \\
\hline GOV 1 & $1452(63.1)$ & $1437(63.4)$ & $1425(63.6)$ \\
\hline GOV 2 & $507(22.1)$ & $495(21.8)$ & $486(21.7)$ \\
\hline IGV 1 & $340(14.8)$ & $336(14.8)$ & $329(14.6)$ \\
\hline Total & 2299 & 2268 & 2240 \\
\hline
\end{tabular}

GOV $1+$ GOV $2=607(30.9 \%)$

GOV $2+\operatorname{IGV} 1=13(1.5 \%)$

GOV $1+\operatorname{IGV} 1=382(21.3 \%)$

GOV $1+\operatorname{GOV} 2+\operatorname{IGV} 1=378(16.4 \%)$

and endoscopic ultrasound (EUS)-guided coil and glue 7/18 (31.8\%). All patients had control of bleed. Two patients in the TIPSS group had refractory encephalopathy and later succumbed to pneumonia ( - Table 5 ).

Before the second session, 31 (1.3\%) patients were lost to follow-up. Mean number of glue injections needed to obliterate residual gastric varices was $1.6(\times 0.5 \mathrm{~mL})$ (range 0 to 7). Variceal obturation was noted in 2220 (97.9\%) cases. Glue extrusion with ulcers was noted in 145 (6.4\%) cases. Rebleed before the second session was noted in $48(2.1 \%)$ cases. Rebleed was most common among IGV1 (63\%), followed by GOV2 (32\%) and GOV1 (5\%). There was no difference between F2 and F3 among IGV1 that rebled (-Table 6).

Before the third session, 281 (1.2\%) patients were lost to follow-up. Average amount of glue used in this session for obliteration of residual gastric varices was $1.2(\times 0.5 \mathrm{~mL})$. Variceal obturation was noted in 2225 (99.3\%) cases. Glue extrusion with ulceration was noted in 90 (4.3\%) cases. Recurrent bleed between and second and third session was noted in $15(0.7 \%)$ cases. It was $13(0.7 \%)$ in IGV1, $2(0.1 \%)$ in GOV2, and 0 in GOV1. ( - Table 7 ).

We encountered a few complications in our series. Pulmonary aspiration of blood was noted in $21(0.9 \%)$ cases.
Table 3 Size of IGV varices

\begin{tabular}{|l|l|}
\hline F1 & $28(8.2 \%)$ \\
\hline F2 & $149(43.8 \%)$ \\
\hline F3 & $163(47.9 \%)$ \\
\hline
\end{tabular}

Table 4 Procedure-related outcome of session 1-initial bleeding episode

\begin{tabular}{|l|l|}
\hline Mean number of glue & 3.6 (range 0-16) \\
\hline Variables & $N(\%)$ \\
\hline Hemostasis & $2281(99.2)$ \\
\hline Endotracheal intubation & $333(14.5)$ \\
\hline Reasons & \\
\hline 1. Active bleeding & $177(53.1)$ \\
\hline 2. Encephalopathy & $135(40.5)$ \\
\hline 3. Respiratory distress & $22(6.6)$ \\
\hline Blood in stomach & $945(41.1)$ \\
\hline Active spurting & $234(10.2)$ \\
\hline Uncontrolled bleeding & $18(0.8)$ \\
\hline
\end{tabular}

Total patients- 2299

Table 5 Management of uncontrolled bleed in session 1

\begin{tabular}{|c|l|}
\hline Uncontrolled bleeding & $18(0.8)$ \\
\hline Hepatocellular carcinoma & $10 / 18(55.6)$ \\
\hline Management & \\
\hline TIPSS & $11 / 18(61.1)$ overall $(0.5)$ \\
\hline EUS-guided coiling & $7 / 18(38.8)$ overall $(0.3)$ \\
\hline
\end{tabular}

Abbreviations: EUS, endoscopic ultrasound; TIPSS, transjugular intrahepatic portosystemic shunt.

Bleeding from ulcers secondary to glue injections were noted in $63(2.8 \%)$ cases. We encountered $4(0.17 \%)$ procedure-related deaths, all postoperatively in 48 hours. None was due to pulmonary embolism. Three $(0.13 \%)$ were due to advanced liver disease and multiorgan failure and $1(0.04 \%)$ was due to cardiac arrest. Echocardiography of this patient did not reveal pulmonary embolism (-Table 8).

Glue splashing into the operator's eyes was encountered twice during the early cases. Immediate thorough lavage with normal saline and continuous methyl cellulose lubricant eye drops was used for management without any severe adverse effect.

Three incidents of needle getting stuck in the varix were noted. We pulled out the sheath with mild force. This led to the needle being separated from the sheath, leaving the needle in the varix. No attempt was made to remove the needle from the glue cast. On follow-up, the needle was also extruded along with glue cast. No bowel perforation or injury was noted in any of the three patients.

\section{Discussion}

Glue has been studied before and compared with different modalities for management of gastric varices. Initial 
Table 6 Outcomes of session 2: after 4 to 6 weeks

\begin{tabular}{|l|l|l|l|}
\hline & $\begin{array}{l}\text { Glue Extrusion with ulcers } \\
N(\%)\end{array}$ & $\begin{array}{l}\text { Only thrombosis without ulcers } \\
N(\%)\end{array}$ & $\begin{array}{l}\text { Rebleed } \\
N(\%)\end{array}$ \\
\hline GOV 1 & $6(0.5)$ & $1,430(63.1)$ & $1(0.04)$ \\
\hline GOV 2 & $28(1.2)$ & $454(20.0)$ & $13(0.6)$ \\
\hline IGV 1 & $111(4.9)$ & $191(8.4)$ & $34(1.5)$ \\
\hline Total & $145(6.4 \%)$ & $2,075(91.5)$ & $48(2.1)$ \\
\hline
\end{tabular}

Patients lost to follow-up-31 (1.3\%).

Total patients-2268.

Mean number of glue: 1.6 (range $0-7$ ).

Table 7 Outcome of session 3: after 3 months to 6 months

\begin{tabular}{|l|l|l|l|}
\hline & $\begin{array}{l}\text { Glue extrusion with ulcers } \\
N(\%)\end{array}$ & $\begin{array}{l}\text { Only thrombosis without ulcers } \\
N(\%)\end{array}$ & $\begin{array}{l}\text { Rebleed } \\
N(\%)\end{array}$ \\
\hline GOV 1 & $2(0.09)$ & $1,423(63.5)$ & $0(0)$ \\
\hline GOV 2 & $15(0.7)$ & $469(20.9)$ & $2(0.09)$ \\
\hline IGV 1 & $73(3.3)$ & $243(10.8)$ & $13(0.6)$ \\
\hline Total & $90(4.0)$ & $2,135(95.3)$ & $15(0.7)$ \\
\hline
\end{tabular}

Patients lost to follow up-28 (1.2\%).

Total patients-2,240.

Mean number of glue: 1.2 (range $0-3$ ).

Table 8 Adverse events

\begin{tabular}{|l|l|}
\hline Variables & $N(\%)$ \\
\hline Aspiration & $21(0.9)$ \\
\hline Bleeding from glue ulcers & $63(2.8)$ \\
\hline Death Postoperative & $4(0.17)$ \\
\hline Advanced liver disease & $3(0.13)$ \\
\hline Cardiac arrest & $1(0.04)$ \\
\hline
\end{tabular}

hemostasis was similar or better with glue as compared with alcohol, ${ }^{4}$ ethanolamine oleate ${ }^{5}$ and band ligation. ${ }^{6}$ Glue was found to be better than alcohol, ${ }^{4}$ ethanolamine oleate ${ }^{5}$ and band ligation $^{6}$ for prevention of rebleed. Two retrospective studies compared cyanoacrylate injection with TIPSS for bleeding gastric varices. There was no difference in rebleeding rates at 72 hours, 3 months, and 1 year, and no difference in 3-month survival. Cyanoacrylate therapy had a higher 30-day rebleed rate than TIPSS. 7,8

By our protocol, we achieved hemostasis in 99.2\% cases with gastric variceal bleed. This was comparable to available data (91 to $100 \%$-sample size 1234,7 one case series and 6 retrospective studies). ${ }^{9}$ The amount of cyanoacrylate glue needed varied from one to sixteen. We injected as many glues as needed for complete variceal obturation, without any upper cutoff limit. No clinical evidence of pulmonary embolism was noted in our study despite using large number of glue injections, which is less compared with available data $(0.5-4.3 \%) .{ }^{10}$ Gotlib et al did not encounter pulmonary embolism in 317 patients over 5 years. ${ }^{11}$ This is attributed to not using any lipiodol in any of the cases. We achieved immediate solidification of all varices with our technique, making sure we had a visible bulge, which felt hard on palpation with needle sheath. Glue extrusion with ulcers was seen in $6.4 \%$, which was comparable with existing literature (4.4\% Cheng et al, 6.2\% Wang et al).,12

Major complications in previous literature ranged from 0 to $8 \%$. Despite having no volume restriction for amount of glue per varix and using increased amount of distilled water, we did not encounter clinical evidence of pulmonary embolism. Previous studies suggest higher risk of glue embolism when amount of distilled water injected exceeds dead space in the catheter. This is contrary to our findings. This might be due to the use of lipiodol diluted glue in previous studies ${ }^{13,14}$ and undiluted glue in ours. Kumar et al used $2 \mathrm{~mL}$ flush after injecting undiluted glue without any complications. ${ }^{1}$ Using more amount of distilled water decreases chances of needle impaction in the glue cast in our experience. This needs to be confirmed in further studies. Instead of restricting the amount of glue per session, aiming for complete variceal obturation in each session appears to be safe as seen from similar or lesser rebleed in our data as compared with existing literature. Comparative studies are however required.

Bleeding was not controlled in only $0.8 \%$ of our patients in spite of all attempts. The cases were controlled by subjecting 11 of these patients to TIPSS, but recently we have started using coils and glue, which we used successfully in seven of our patients in the last few years.

In follow-up of our cases, we found that ulcerations secondary to glue extrusion were noted in $6.4 \%$ after 4 to 6 weeks of first session and $4.3 \%$ after 3 to 4 months of the second session. Wang et al reported glue extrusion with ulceration in $8.1 \% .^{12}$ Rebleed from these ulcers was noted in $2.1 \%$ after the first session and $0.7 \%$ after the second session which was much lower than previous literature (7-28\% and 4.4-8.1\%, respectively). The rebleed in these cases was controlled by 
injecting a feeding vessel in the vicinity of the ulcer which worked in almost all cases.

We encountered four deaths within 48 hours of procedure in our series. Three were due to advanced liver disease and one had a cardiac event postoperatively due to impaired cardiac output. CT pulmonary angiography in all four patients did not show pulmonary embolism. Previous studies showed higher incidence of embolic complications when large volume of glue or distilled water was used. These studies however used diluted glue (with lipiodol) and not undiluted glue. Kumar et al used $2 \mathrm{~mL}$ distilled water without any evidence of clinical distal embolism. ${ }^{1}$

We used glue even for GOV1. There is ample data on use of glue in GOV1. Comparative studies (GVL vs. glue) for GOV1 are also available with results in favor of glue. Baveno VI guidelines suggest either EVL or glue for management of GOV 1. Cochrane metanalysis of three randomized control trials (RCTs) showed no difference in initial hemostasis between EVL and glue. This metanalysis was dominated by a single large study which included only GOV1. ${ }^{15}$ Lo et al showed similar initial hemostasis but higher rebleed and mortality with EVL for GOV1 as compared with glue. This was, however, not an RCT. ${ }^{6}$ There has been discrepancy in rebleeding rates among type of varices treated by glue, but all studies show lower rebleed rates with GOV1.,6,16,17

One of the limitations of this study is retrospective data analysis and only short-term follow-up. A fair number of patients were not available in the long run for a robust longterm follow-up, so we have not included the data beyond three sessions.

\section{Conclusion}

Hence, we can conclude from our experience that glue is an excellent modality for management of gastric varices (GOV 1, 2 and IGV) Amount of glue per session should not be a restricting factor, using as much amount of glue as needed to ensure a hemostasis and achieve complete obliteration of varices in the long run. Using small glue bolus with a more amount of distilled water ensures complete filling of the varix, causes less ulcerations, less rebleed and more fibrosis, and we have fewer complications like pulmonary aspiration and very low incidence of death. However, more RCTs are necessary to confirm our findings. Avoiding lipiodol is the key to preventing pulmonary embolism.

\section{Conflicts of Interest}

None declared.

\section{References}

1 Kumar A, Singh S, Madan K, Garg PK, Acharya SK. Undiluted $\mathrm{N}$-butyl cyanoacrylate is safe and effective for gastric variceal bleeding. Gastrointest Endosc 2010;72(4):721-727
2 Cheng L-F, Wang Z-Q Li C-Z, Lin W, Yeo AET, Jin B. Low incidence of complications from endoscopic gastric variceal obturation with butyl cyanoacrylate. Clin Gastroenterol Hepatol 2010;8(9):760-766

3 Garcia-Tsao G, Abraldes JG, Berzigotti A, Bosch J. Portal hypertensive bleeding in cirrhosis: Risk stratification, diagnosis, and management: 2016 practice guidance by the American Association for the study of liver diseases. Hepatology 2017;65(1):310-335

4 Sarin SK, Jain AK, Jain M, Gupta R. A randomized controlled trial of cyanoacrylate versus alcohol injection in patients with isolated fundic varices. Am J Gastroenterol 2002;97(4):1010-1015

5 Oho K, Iwao T, Sumino M, Toyonaga A, Tanikawa K. Ethanolamine oleate versus butyl cyanoacrylate for bleeding gastric varices: a nonrandomized study. Endoscopy 1995;27(5):349-354

6 Lo GH, Lai KH, Cheng JS, Chen MH, Chiang HT. A prospective, randomized trial of butyl cyanoacrylate injection versus band ligation in the management of bleeding gastric varices. Hepatology 2001;33(5):1060-1064

7 Procaccini NJ, Al-Osaimi AMS, Northup P, Argo C, Caldwell SH. Endoscopic cyanoacrylate versus transjugular intrahepatic portosystemic shunt for gastric variceal bleeding: a single-center U.S. analysis. Gastrointest Endosc 2009;70(5):881-887

8 Tan P-C, Hou M-C, Lin H-C, et al. A randomized trial of endoscopic treatment of acute gastric variceal hemorrhage: $\mathrm{N}$-butyl-2-cyanoacrylate injection versus band ligation. Hepatology 2006;43(4):690-697

9 Sarin SK, Lahoti D, Saxena SP, Murthy NS, Makwana UK. Prevalence, classification and natural history of gastric varices: a long-term follow-up study in 568 portal hypertension patients. Hepatology 1992;16(6):1343-1349

10 Saraswat VA, Verma A. Gluing gastric varices in 2012: lessons learnt over 25 years. J Clin Exp Hepatol 2012;2(1):55-69

11 Gotlib J-P. Endoscopic obturation of esophageal and gastric varices with a cyanoacrylic tissue adhesive. Can J Gastroenterol 1990;4:637-638

12 Wang Y-M, Cheng L-F, Li N, Wu K, Zhai J-S, Wang Y-W. Study of glue extrusion after endoscopic N-butyl-2-cyanoacrylate injection on gastric variceal bleeding. World J Gastroenterol 2009;15(39):4945-4951

13 Seewald S, Ang TL, Imazu H, et al. A standardized injection technique and regimen ensures success and safety of N-butyl2-cyanoacrylate injection for the treatment of gastric fundal varices (with videos) Gastrointest Endosc 2008;68(3):447-454

14 Seewald S, Sriram PVJ, Naga M, et al. Cyanoacrylate glue in gastric variceal bleeding. Endoscopy 2002;34(11):926-932

15 Ríos Castellanos E, Seron P, Gisbert JP, Bonfill Cosp X. Endoscopic injection of cyanoacrylate glue versus other endoscopic procedures for acute bleeding gastric varices in people with portal hypertension. Cochrane Database Syst Rev 2015; (5):CD010180

16 Kind R, Guglielmi A, Rodella L, et al. Bucrylate treatment of bleeding gastric varices: 12 years' experience. Endoscopy 2000;32(7):512-519

17 Lee HA, Chang JM, Goh HG, et al; Korean Portal Hypertension Study Group. Prognosis of patients with gastric variceal bleeding after endoscopic variceal obturation according to the type of varices. Eur J Gastroenterol Hepatol 2019;31(2):211-217 